Published in final edited form as:

Lancet. 2015 February 28; 385(9970): 792-798. doi:10.1016/S0140-6736(14)62052-3.

\title{
EXTENDED DURATION DUAL ANTIPLATELET THERAPY AND MORTALITY - A SYSTEMATIC REVIEW AND META-ANALYSIS
}

\author{
Sammy Elmariah, MD, MPH, \\ Cardiology Division, Department of Medicine, Massachusetts General Hospital, Harvard Medical \\ School, Boston, MA, USA \\ Harvard Clinical Research Institute, Boston, MA, USA \\ Laura Mauri, MD, MSc, \\ Harvard Clinical Research Institute, Boston, MA, USA \\ Division of Cardiovascular Medicine, Department of Medicine, Brigham and Women's Hospital, \\ Harvard Medical School, Boston, MA, USA \\ Gheorghe Doros, PhD, \\ Harvard Clinical Research Institute, Boston, MA, USA \\ Department of Biostatistics, Boston University School of Public Health, Boston, MA, USA

\section{Benjamin Z. Galper, MD, MPH,} \\ Division of Cardiovascular Medicine, Department of Medicine, Brigham and Women's Hospital, \\ Harvard Medical School, Boston, MA, USA
}

Kelly E. O'Neill,

Division of Cardiovascular Medicine, Department of Medicine, Brigham and Women's Hospital, Harvard Medical School, Boston, MA, USA

\section{Philippe Gabriel Steg, MD,}

Université Paris-Diderot, Sorbonne Paris-Cité, Paris, France

Département Hospitalo-Universitaire FIRE (Fibrosis, Inflammation and Remodeling), INSERM U-1148 and Hôpital Bichat, Assistance Publique-Hôpitaux de Paris, Paris, France

National Heart Lung Institute, Imperial College, Royal Brompton Hospital, London, United Kingdom

Dean J. Kereiakes, MD, and

The Lindner Research Center, The Christ Hospital Heart and Vascular Center, Cincinnati, OH, USA

Robert W. Yeh, MD, MSc

Corresponding author: Robert W. Yeh, MD, MSc, Gray-Bigelow 800, 55 Fruit Street, Boston, MA 02114, Tel: 617-643-7357, Fax: 617-726-7419, ryeh@mgh.harvard.edu.

Contributors

SE, LM, and RWY contributed to the study conception and design. SE, BZG, KEO executed the systematic literature search and selected studies for inclusion. GD performed statistical analyses and generated figures. SE, LM, GD, and RWY interpreted data. SE and RWY wrote the first draft of the manuscript. All authors critically revised the manuscript for important intellectual content and approved the final draft. 
Cardiology Division, Department of Medicine, Massachusetts General Hospital, Harvard Medical

School, Boston, MA, USA

Harvard Clinical Research Institute, Boston, MA, USA

\section{Abstract}

Background-Treatment with the combination of aspirin and a P2Y12 inhibitor is commonly employed in a number of cardiovascular conditions. The overall impact of such treatment on allcause mortality is unknown. In the Dual Antiplatelet Therapy (DAPT) Study, an international multicenter randomized placebo-controlled trial comparing 30 versus 12 months of dual antiplatelet therapy after coronary stenting, continuation of DAPT beyond 12 months was associated with an increase in mortality at trial completion, largely attributable to an increase in non-cardiovascular death. Given the potential public health importance of this, we performed a meta-analysis of all randomized, controlled trials of DAPT duration across a wide array of cardiovascular conditions to evaluate the impact of extended duration DAPT on mortality.

Methods-A systematic literature search of MEDLINE, Embase, and Cochrane Central Register of Controlled Trials (CENTRAL) database was performed to identify randomized controlled trials evaluating the impact of extended duration versus no or short-duration DAPT. Study results were pooled using a hierarchical Bayesian random-effects model. The primary outcomes examined were hazard ratios comparing rates of all-cause, cardiovascular, and non-cardiovascular death.

Findings-Including the DAPT Study, we identified 14 eligible trials that randomized 69,644 subjects to different durations of DAPT. Compared with aspirin alone or short-duration DAPT ( $\leq$ 6 months), continued DAPT was not associated with a difference in all-cause mortality (hazard ratio $[\mathrm{HR}] 1 \cdot 05 ; 95 \%$ credible interval [CrI], 0.96-1.19). Similarly, cardiovascular (HR 1.01; 95\% CrI, 0.93-1.12) and non-cardiovascular mortality (HR 1.04; 95\% CrI, 0.90-1.26) were no different with extended duration vs. short duration DAPT or aspirin alone.

Interpretation-In a meta-analysis of 14 trials, extended duration DAPT was not associated with a difference in the risk of all-cause, cardiovascular, or non-cardiovascular death compared with aspirin alone or short duration DAPT.

\section{INTRODUCTION}

Treatment with dual antiplatelet therapy (DAPT) using a combination of aspirin and a P2Y12 inhibitor is widely used for the prevention of ischemic complications associated with a number of cardiovascular diseases, including peripheral arterial, cerebrovascular, and coronary artery disease. In each of these areas, the duration of therapy that best balances the benefits and risks of DAPT is uncertain. In patients at high risk for ischemic events, treatment with DAPT may prevent potentially fatal thrombotic events. However, risks of adverse events associated with long-term DAPT also exist, primarily mediated through bleeding, and could outweigh benefits, such that overall mortality is unchanged or even increased.

Prior studies evaluating the impact of extended duration DAPT on mortality have varied. The Clopidogrel and Aspirin versus Aspirin Alone for the Prevention of Atherothrombotic 
Events (CHARISMA) Trial showed no difference in all-cause mortality among a heterogeneous population with or at risk for cardiovascular disease treated with DAPT versus aspirin alone. ${ }^{1}$ On the other hand, the Secondary Prevention of Small Subcortical Strokes (SPS3) Trial showed a statistically significant increase in mortality associated with DAPT compared to aspirin alone among patients with recent lacunar infarcts, an unexpected finding that was attributed to either the specific population enrolled or to chance. ${ }^{2}$ Most recently, the Dual Antiplatelet Therapy (DAPT) Study, an international multicenter randomized double placebo controlled trial that compared 30 versus 12 months of DAPT after percutaneous coronary intervention (PCI) with stents, showed that continuation of DAPT beyond 12 months in DES-treated subjects was associated with an increase in the prespecified secondary endpoint of total mortality at trial completion, a difference driven primarily by an increase in non-cardiovascular death. ${ }^{3}$

If true, an increase in non-cardiovascular and all-cause mortality due to extended treatment with DAPT would have an important impact on the many cardiovascular patients treated with these agents each year. We therefore conducted a comprehensive meta-analysis of randomized controlled trials evaluating the impact of extended duration DAPT on all-cause, cardiovascular and non-cardiovascular mortality.

\section{METHODS}

\section{Data Sources and Search Strategy}

We conducted a systematic review and meta-analysis in accordance with the Preferred Reporting Items for Systematic Reviews and Meta-Analyses (PRISMA) statement for reporting systematic reviews and meta-analyses of healthcare interventions to examine the impact of extended duration DAPT on mortality. ${ }^{4}$ A systematic literature search of MEDLINE (using the PubMed interface), Embase, and Cochrane Central Register of Controlled Trials (CENTRAL) databases was performed for relevant randomized clinical trials published prior to October 1, 2014. The following search terms were used: "clopidogrel", "Plavix", "prasugrel”, "Effient”, "ticagrelor", "Brilinta", "thienopyridine”, "dual antiplatelet therapy", "DAPT", "death", "mortality", "survival", "randomized controlled trial", "random", "random allocation", "double-blind", and "single-blind". References of identified studies were also manually searched. Searches were restricted to trials of human subjects with full text published in English. The systematic search strategy is outlined in the Appendix (See Study Protocol). In addition to these studies, we also included results of for all subjects randomized in the DAPT Study (BMS- and DES-treated subjects), which was not published at the time of this analysis.

\section{Study Selection and Endpoints}

Three investigators independently executed the systematic search and critically reviewed identified studies in order to ensure that each satisfied the following criteria: 1) subjects within the study were randomly assigned to receive either extended duration DAPT, defined as aspirin and co-administration of a P2Y12 receptor inhibitor (clopidogrel, prasugrel, or ticagrelor) for at least 6 months after randomization, or no or short duration DAPT, defined as aspirin alone or DAPT for less than 6 months after randomization; 2) all-cause, 
cardiovascular, and/or non-cardiovascular mortality was reported at 1-year follow-up or later; and 3) the study included >100 subjects. We classified all deaths labeled as "cardiovascular", "cardiac", or "vascular" by the individual trials as cardiovascular deaths for this meta-analysis, and all other deaths to be non-cardiovascular. In trials that did not specifically report non-cardiovascular mortality, but did report total and cardiovascular mortality rates, non-cardiovascular death rates were arithmetically generated. Subgroup analyses were excluded, and for each trial, the manuscript reporting the longest follow-up included. For trials in which there was a known period of observation in which DAPT treatment was not different between study arms, we included the longest duration of follow up that preceded this period. Data extraction and assessment for risk of bias were independently performed by two investigators using the Cochrane Collaboration's tool for assessing risk of bias ${ }^{5}$ and checked for consistency by a third investigator. Discrepancies in study selection were resolved by consensus.

\section{Statistical Analysis}

With survival-type data log hazard ratio and its variance has been recommended as an appropriate summary statistics for meta-analyses. ${ }^{6}$ This measure allows for censoring and accommodates variable length of follow-up for each of the included trials. Hazard ratios (HRs) with 95\% confidence intervals for all-cause, cardiovascular, and non-cardiovascular mortality with extended versus short duration DAPT were extracted for each of the trials. For studies in which the HR and the associated 95\% confidence interval were reported, the logarithm of HR and the associated variance were calculated. For studies in which HR was not reported for an endpoint, the $\log (\mathrm{HR})$ and its variance were estimated using a previously validated method, as follows: 6,7

$\log -\mathrm{HR}=2 *[(\#$ observed events Group 1) $-(\#$ observed events Group 2) $] /[(\#$ observed events Group 1) + (\# observed events Group 2)]

Variance $(\log -\mathrm{HR})=4 /$ [(\# observed events Group 1) $+(\#$ observed events Group 2) $]$.

This approximation is valid for balanced, large studies with similar follow-up between treatment groups.

To provide a broad sample of data relevant to the association between extended duration DAPT and mortality, we included studies that were heterogeneous with respect to trial methods, study populations, and follow-up durations. However, all studies included compared extended duration to short duration DAPT, reported all-cause mortality, and adjudicated cardiovascular causes of death. To accommodate the heterogeneity across studies, we used random-effects meta-analysis to synthesize results. In particular, we used a Bayesian hierarchical model to estimate the random effects model. Potential advantages of a Bayesian approach, including the appropriate reflection of the uncertainty in estimates of hyperparameters, have been previously described. ${ }^{8}$ The open-source program OpenBUGS (Bayesian inference using Gibbs Sampling) was used to fit Markov Chain Monte Carlo (MCMC) models. ${ }^{9}$ Posterior inferences (HR and 95\% credible intervals [CrI]) were calculated by sampling from the posterior distribution of the parameters. We used noninformative priors (normal distribution with mean $=0$, standard deviation $=1000$ ) for the overall mean HR and inverse-gamma $(0 \cdot 00001,0.00001)$ for the between study variance. 
Heterogeneity across studies was assessed using the Cochran Q test and the $\mathrm{I}^{2}$ statistic. ${ }^{10}$ Funnel plots were created to assess for bias across studies.

We conducted sensitivity analyses to investigate the robustness of our results to various assumptions. First, we repeated our analysis omitting one study at a time to assess whether any of the included studies had a large influence on the results. Second, we restricted the analysis to studies specific to coronary artery disease (e.g. acute coronary syndrome and/or PCI). Additionally, a sensitivity analysis was conducted to study the robustness of our choice of prior distribution. Finally, to evaluate whether duration of exposure to DAPT was related to the magnitude of a possible treatment effect of DAPT on mortality across trials, we performed a meta-regression according to difference in duration of DAPT exposure between study arms. Each of these sensitivity analyses was performed for the endpoints of all-cause, cardiovascular and non-cardiovascular mortality. All primary and sensitivity analyses were pre-specified, apart from the analysis limited to patients with coronary artery disease, which was performed in a post-hoc manner. The pre-specified study protocol can be found in the Appendix (See Study Protocol).

All analyses were performed at the Harvard Clinical Research Institute. Institutional review board approval was not obtained due to the nature of the study.

\section{Role of the funding source}

There was no funding source for this study. All authors had full access to all the data used in the study, and Dr. Yeh had final responsibility for the decision to submit for publication.

\section{RESULTS}

\section{Study Selection}

Our systematic literature search identified 2,456 articles, of which 13 met the inclusion criteria for this analysis (figure 1). After adding results of the DAPT Study, 14 studies comprising 69,644 subjects were included in our meta-analysis. ${ }^{1-3,11-21}$ The subject populations enrolled in the trials were as follows: ten trials of subjects with coronary artery disease after percutaneous coronary intervention and/or acute coronary syndrome $(1,344$ deaths, 42,606 subjects), 3,12,14-21 one trial of subjects who underwent surgical revascularization for peripheral arterial disease (41 deaths, 851 subjects), ${ }^{11}$ one trial of subjects with recent lacunar stroke (190 deaths, 3,020 subjects), ${ }^{2}$ one trial of subjects at high risk of atherothrombotic events (745 deaths, 15,603 subjects), ${ }^{1}$ and one trial of subjects with atrial fibrillation (1,666 deaths, 7,554 subjects; table 1). ${ }^{13}$ All trials were multicenter, randomized, controlled trials with low risk of bias (Appendix, Supplemental Table 5).

Dual antiplatelet therapy in all included trials was comprised of aspirin and thienopyridine. ${ }^{1-3,11-21}$ All trials evaluated clopidogrel as the sole thienopyridine apart from ARCTIC-Interruption and the DAPT Study, which included both clopidogrel- and prasugreltreated patients (prasugrel use 32\% among randomized BMS- and DES-treated subjects in the DAPT Study, and 9\% among randomized subjects in ARTIC-Interruption). ${ }^{2,22}$ Eight trials compared DAPT to aspirin alone (55,198 subjects), ${ }^{1-3,11-13,20,21}$ of which three trials, DES-LATE, ARCTIC-Interruption, and the DAPT Study compared the continuation of 
DAPT beyond 12 months to aspirin alone in subjects having already received 12 months or more of DAPT. $3,20,21$ The remaining six trials compared extended duration DAPT to short duration DAPT (12,102 subjects). ${ }^{12,14-18}$ The difference in DAPT duration between study arms in all 14 trials ranged from six months to a median of $43 \cdot 2$ months.

\section{Meta-Analysis of Prolonged DAPT and Mortality}

All 14 studies reported all-cause mortality. There was no significance evidence of heterogeneity among trials as assessed by Cochran's $\mathrm{Q}(\mathrm{Q}=17 \cdot 7, \mathrm{p}=0 \cdot 17)$, and the $\mathrm{I}^{2}$ value was $26.5 \%$. In a Bayesian random effects model, extended duration DAPT was not associated with all-cause mortality when compared to short-duration DAPT or aspirin alone (HR 1.05; CrI, 0.96-1.19; figure 2). The overall effect size was comparable to that seen in all trials prior to the DAPT Study (HR 1.03; 95\% CrI, 0.94-1·16).

Cardiovascular and non-cardiovascular mortality rates were available for $12(63,915)$ and 11 (63,064 subjects) studies, respectively. In a Bayesian random effects meta-analysis, extended duration DAPT was not associated with cardiovascular mortality (HR 1.01; 95\% CrI, 0.93-1.12; figure 3A) or non-cardiovascular mortality (HR 1.04; CrI, 0.90-1.26; figure 3B). These results were consistent both before and after inclusion of the DAPT Study results. Funnel plots suggested no evidence of publication bias. (Appendix, Supplemental Figure 1)

\section{Sensitivity Analyses}

Sequential exclusion of each trial from the meta-analysis showed results consistent with the full analysis for all-cause, cardiovascular, or non-cardiovascular mortality (Appendix, Supplemental Figure 2). Restriction of the analysis to the ten trials (42,606 subjects) exclusively enrolling subjects treated for coronary artery disease was consistent with the full analysis (restricted analysis: HR 1.05; 95\% CrI, 0.92-1.24; Supplemental Figure 3). Similarly, results of restricted analyses in the eight coronary artery disease trials $(39,231$ subjects) reporting cardiovascular (HR 0.98; 95\% CrI, 0.84-1.17; Supplemental Figure 4) and non-cardiovascular (HR 1.22; 95\% CrI, 0.92-1.61; Supplemental Figure 5) mortality were consistent with the full analysis, Finally, meta-regression did not demonstrate a significant effect of DAPT treatment duration difference on HR for all-cause, cardiovascular, or non-cardiovascular mortality across studies (Appendix, Supplemental Figures $6-8)$.

\section{DISCUSSION}

In this systematic review and meta-analysis of more than 67,000 subjects included in 14 randomized trials, with average follow up of 22 months, we did not detect an association between extended duration DAPT and all-cause, cardiovascular and non-cardiovascular mortality. The meta-analysis findings were not sensitive to the exclusion of any single trial, and while the enrolled populations varied, there was no substantial heterogeneity in trial results with respect to the relationship of DAPT to mortality.

The evaluation of extended duration DAPT in cardiovascular patients has been primarily focused on examining the tradeoff between ischemic complications (myocardial infarction, 
stent thrombosis, and stroke) and bleeding complications associated with more potent antiplatelet therapy. The DAPT Study, which compared 30 months vs. 12 months of thienopyridine in aspirin-treated subjects undergoing PCI with stent placement, found significant reductions in stent thrombosis and the combination of death, myocardial infarction and stroke at 30 months in patients treated with extended thienopyridine. Unexpectedly, however, all-cause mortality was numerically higher in DES-treated subjects randomized to continued thienopyridine (HR 1.36; 95\% CI, 1.00-1.85 at 30 months, and HR 1.36 ; $95 \%$ CI, $1.02-1.82$ at the 33 months). These differences were driven by differences in non-cardiovascular mortality (HR 2.23; 95\% CI, 1.32-3.78 at 30 months). A blinded independent review of cases of death in the DAPT Study found that bleeding events did not occur in the majority of non-cardiovascular deaths and did not account for the numerical difference in deaths between arms. ${ }^{3}$ Similar findings had previously been observed in the SPS3 trial, a study of patients with recent lacunar stroke, and had been attributed to either a specific effect in the enrolled population or to chance. ${ }^{2}$

In light of the millions of patients treated with thienopyridines, including the more than 1 million undergoing PCI with stents each year worldwide, ${ }^{23}$ a true increase in mortality associated with extended thienopyridine use among the diverse population of cardiovascular patients could have profound public health consequences. Conversely, widespread fear over the risks of continued thienopyridine use could results in inappropriate discontinuation, leading to an increased risk of ischemic events. ${ }^{24,25}$ This is particularly important in light of the renewed observation within the DAPT trial that even late planned thienopyridine discontinuation 30 months after PCI was associated with a detectable increase in risk of ischemic events. Therefore, it was imperative to examine the impact of extended treatment with DAPT on mortality among a wide group of cardiovascular patients, to help frame trialspecific results against of the larger body of prior literature evaluating DAPT duration.

We did not detect a significant increase or reduction in mortality with extended DAPT treatment for the broad population of cardiovascular patients represented in randomized trials to date. While these results provide reassurance that there may not be a substantial and generalized hazard of long-term DAPT therapy for mortality, they do not exclude the possibility of small treatment effects that even this large metanalysis may not have been powered to detect, or effects within particular subgroups. For example, in the setting of coronary stent treatment, DAPT beyond 12 months is associated with reduction in myocardial infarction and stent thrombosis, each of which may be fatal. However, with improved recognition and treatment of these conditions, the associated mortality of these ischemic events may be less than in prior eras, and the relative contributions of noncardiovascular causes of death represent a growing proportion of mortality. ${ }^{26,27}$ In light of the neutral association with mortality, and the offsetting ischemic benefits and bleeding risks, the decision to continue DAPT beyond what is currently recommended in guidelines should be tailored to the specific anticipated risks for individual patients.

Our study has a number of important limitations. First, the studies included in the metaanalysis enrolled heterogeneous populations, had differing study protocols and endpoint definitions, and compared different durations of DAPT. Although study populations differed, when limiting trials to those that enrolled only subjects with coronary artery 
disease, we found no difference in study results. Despite the size of the overall population considered, the credible intervals for mortality indicate that one cannot definitely rule out a $19 \%$ or less true increase in mortality with extended DAPT vs. control. Furthermore, we did not identify an association between duration of exposure and treatment effects on mortality across studies. Each trial may have reported and adjudicated cardiovascular death slightly differently; however, all trials reported all-cause mortality, which should not be influenced by differences in study definitions or endpoint adjudication. As with any meta-analysis, our study is limited by the limitations of the included studies. While all studies were randomized, not all studies were blinded; however, this also would not be expected to impact the endpoint of mortality substantially. In addition, in all but two trials, clopidogrel was the only P2Y12 inhibitor evaluated, and thus conclusions regarding the association between treatment with other P2Y12 inhibitors and mortality cannot be drawn from this study. The included trials predominantly enrolled patients with coronary artery disease, and the findings may not be generalizable to other patient populations, including high-risk patients who might be excluded from clinical trials. Finally, it was not possible in this triallevel analysis to consider the impact of specific patient characteristics on the association of DAPT with mortality. Future efforts focused on patient-level data are needed in order to thoroughly investigate a potential relationship between extended duration thienopyridine and mortality among selected subgroups of patients.

In summary, in a meta-analysis of 14 trials comparing extended versus short duration DAPT, extended DAPT was not associated with a difference in the risk of all-cause, cardiovascular, or non-cardiovascular death compared with aspirin alone or short duration DAPT. Determining the optimal duration of DAPT in cardiovascular patients should be based on the relative ischemic benefit and bleeding risk of treatment in individual patients.

\section{Supplementary Material}

Refer to Web version on PubMed Central for supplementary material.

\section{Acknowledgements}

There was no funding source for this study.

Declaration of interests

SE reports institutional research grants from the American Heart Association and Siemens Medical Solutions. LM reports institutional research grants from Abbott, Boston Scientific, Cordis, Medtronic, Eli Lilly/Daiichi Sankyo, and sanofi Aventis/Bristol Myers Squibb; and personal fees from Medtronic, Recor, St. Jude Medical, and Biotronik. PGS reports personal fees from Amarin, AstraZeneca, Bayer, Boehringer-Ingelheim, Bristol-MyersSquibb, Daiichi-Sankyo, GlaxoSmithKline, Eli Lilly, Merck-Sharpe-Dohme, Novartis, Otsuka, Pfizer, Roche, Medtronic, sanofi-aventis, Servier, Vivus, Janssn, The Medicines Company, and Orexigen; and grants from sanofiaventis and Servier. Dr. Yeh reports personal fees from Abbott Vascular and Gilead Sciences.

\section{REFERENCES}

1. Bhatt DL, Fox KA, Hacke W, et al. Clopidogrel and aspirin versus aspirin alone for the prevention of atherothrombotic events. The New England journal of medicine. 2006; 354(16):1706-1717. [PubMed: 16531616] 
2. Benavente OR, Hart RG, McClure LA, Szychowski JM, Coffey CS, Pearce LA. Effects of clopidogrel added to aspirin in patients with recent lacunar stroke. The New England journal of medicine. 2012; 367(9):817-825. [PubMed: 22931315]

3. Mauri L, Kereiakes DJ, Yeh RW, et al. Continuation of dual antiplatelet therapy beyond one year after drug-eluting coronary stent procedures. New England journal of medicine. 2014

4. Liberati A, Altman DG, Tetzlaff J, et al. The PRISMA statement for reporting systematic reviews and meta-analyses of studies that evaluate health care interventions: explanation and elaboration. Annals of internal medicine. 2009; 151(4):W65-W94. [PubMed: 19622512]

5. Higgins JP, Altman DG, Gotzsche PC, et al. The Cochrane Collaboration's tool for assessing risk of bias in randomised trials. Bmj. 2011; 343:d5928. [PubMed: 22008217]

6. Parmar MK, Torri V, Stewart L. Extracting summary statistics to perform meta-analyses of the published literature for survival endpoints. Statistics in medicine. 1998; 17(24):2815-2834. [PubMed: 9921604]

7. Tsiatis AA. The asymptomatic joint distribution of the efficient scores test for the proportional hazards model calculated over time. Biometrika. 1981; 68(1):311-315.

8. Spiegelhalter DJ, Myles JP, Jones DR, Abrams KR. Bayesian methods in health technology assessment: a review. Health technology assessment. 2000; 4(38):1-130. [PubMed: 11134920]

9. Lunn D, Spiegelhalter D, Thomas A, Best N. The BUGS project: Evolution, critique and future directions. Statistics in medicine. 2009; 28(25):3049-3067. [PubMed: 19630097]

10. Higgins JP, Thompson SG, Deeks JJ, Altman DG. Measuring inconsistency in meta-analyses. Bmj. 2003; 327(7414):557-560. [PubMed: 12958120]

11. Belch JJ, Dormandy J, Biasi GM, et al. Results of the randomized, placebo-controlled clopidogrel and acetylsalicylic acid in bypass surgery for peripheral arterial disease (CASPAR) trial. Journal of vascular surgery. 2010; 52(4):825-833. 33.e1-33.e2. [PubMed: 20678878]

12. Colombo A, Chieffo A, Frasheri A, et al. Second Generation Drug-Eluting Stents Implantation Followed by Six Versus Twelve-Month - Dual Antiplatelet Therapy- The SECURITY Randomized Clinical Trial. Journal of the American College of Cardiology. 2014

13. Connolly SJ, Pogue J, Hart RG, et al. Effect of clopidogrel added to aspirin in patients with atrial fibrillation. The New England journal of medicine. 2009; 360(20):2066-2078. [PubMed: 19336502]

14. Feres F, Costa RA, Abizaid A, et al. Three vs twelve months of dual antiplatelet therapy after zotarolimus-eluting stents: the OPTIMIZE randomized trial. JAMA : the journal of the American Medical Association. 2013; 310(23):2510-2522.

15. Gwon HC, Hahn JY, Park KW, et al. Six-month versus 12-month dual antiplatelet therapy after implantation of drug-eluting stents: the Efficacy of Xience/Promus Versus Cypher to Reduce Late Loss After Stenting (EXCELLENT) randomized, multicenter study. Circulation. 2012; 125(3): 505-513. [PubMed: 22179532]

16. Kim BK, Hong MK, Shin DH, et al. A new strategy for discontinuation of dual antiplatelet therapy: the RESET Trial (REal Safety and Efficacy of 3-month dual antiplatelet Therapy following Endeavor zotarolimus-eluting stent implantation). Journal of the American College of Cardiology. 2012; 60(15):1340-1348. [PubMed: 22999717]

17. Steinhubl SR, Berger PB, Mann JT 3rd, et al. Early and sustained dual oral antiplatelet therapy following percutaneous coronary intervention: a randomized controlled trial. JAMA : the journal of the American Medical Association. 2002; 288(19):2411-2420.

18. Valgimigli M, Campo G, Monti M, et al. Short- versus long-term duration of dual-antiplatelet therapy after coronary stenting: a randomized multicenter trial. Circulation. 2012; 125(16):20152026. [PubMed: 22438530]

19. Yusuf S, Zhao F, Mehta SR, Chrolavicius S, Tognoni G, Fox KK. Effects of clopidogrel in addition to aspirin in patients with acute coronary syndromes without ST-segment elevation. The New England journal of medicine. 2001; 345(7):494-502. [PubMed: 11519503]

20. Collet JP, Silvain J, Barthelemy O, et al. Dual-antiplatelet treatment beyond 1 year after drugeluting stent implantation (ARCTIC-Interruption): a randomised trial. Lancet. 2014 
21. Lee CW, Ahn JM, Park DW, et al. Optimal duration of dual antiplatelet therapy after drug-eluting stent implantation: a randomized, controlled trial. Circulation. 2014; 129(3):304-312. [PubMed: 24097439]

22. Mauri L, Kereiakes DJ, Yeh RW, et al. DAPT. New England Journal of Medicine. 2014

23. Go AS, Mozaffarian D, Roger VL, et al. Heart disease and stroke statistics--2014 update: a report from the American Heart Association. Circulation. 2014; 129(3):e28-e292. [PubMed: 24352519]

24. Spertus JA, Kettelkamp R, Vance C, et al. Prevalence, predictors, and outcomes of premature discontinuation of thienopyridine therapy after drug-eluting stent placement: results from the PREMIER registry. Circulation. 2006; 113(24):2803-2809. [PubMed: 16769908]

25. Ho PM, Peterson ED, Wang L, et al. Incidence of death and acute myocardial infarction associated with stopping clopidogrel after acute coronary syndrome. JAMA : the journal of the American Medical Association. 2008; 299(5):532-539.

26. Spoon DB, Psaltis PJ, Singh M, et al. Trends in cause of death after percutaneous coronary intervention. Circulation. 2014; 129(12):1286-1294. [PubMed: 24515993]

27. Steg PG, Cheong AP. Death (after percutaneous coronary intervention) is no longer what it used to be. Circulation. 2014; 129(12):1267-1269. [PubMed: 24515992] 


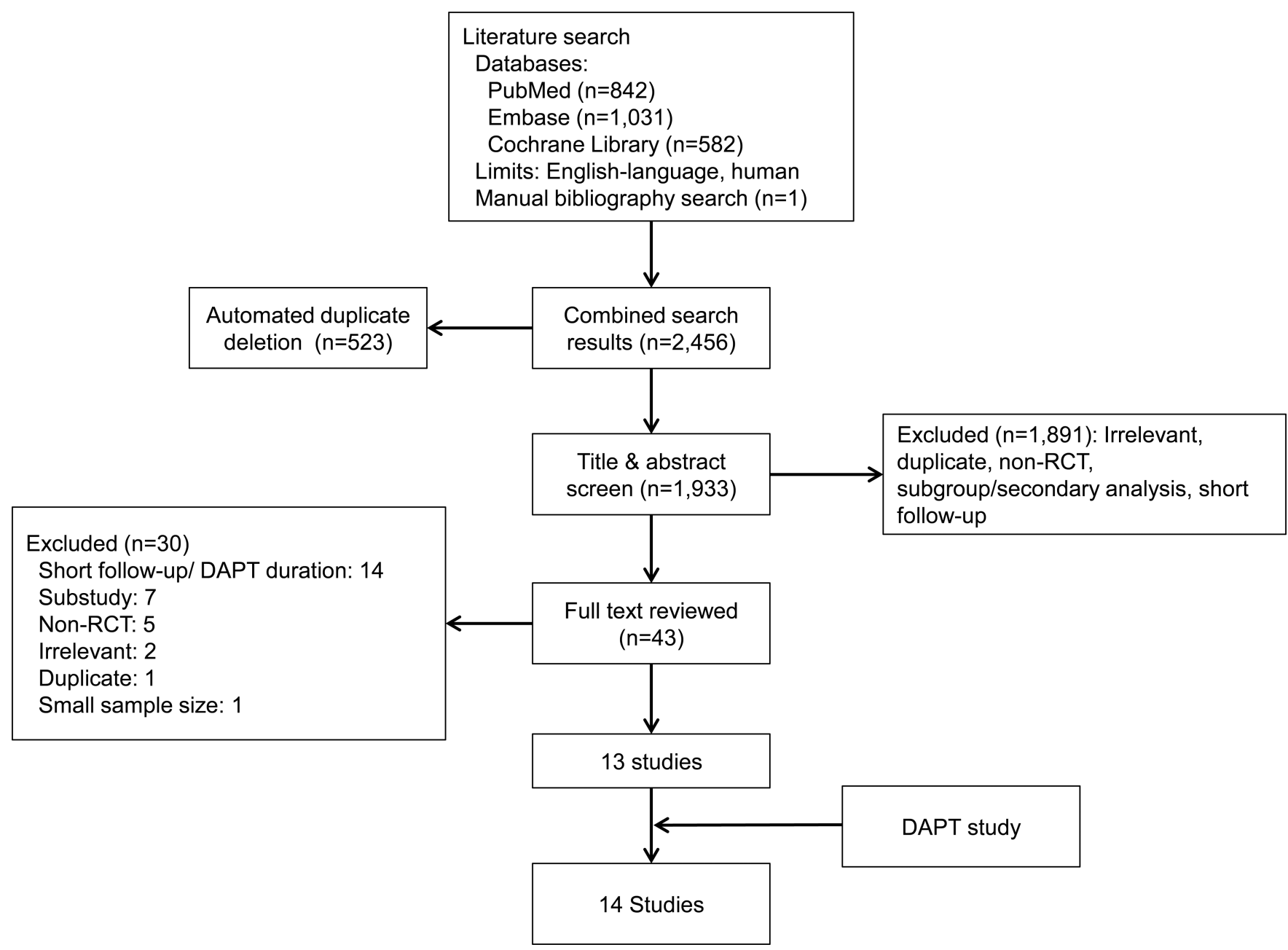

Figure 1. Flow diagram of literature search and study selection 


Study
CASPAR
SPS3
CHARISMA
ACTIVE
OPTIMIZE
EXCELLENT
RESET
CREDO
PRODIGY
CURE
ARCTIC-Interruption
DES LATE
SECURITY
Overall(DAPT NOT Included)
Q = 14.87, $p=0.25 ;$ I2 = 12.6\%
DAPT
Overall(DAPT Included)
$Q=17.68, p=0.17 ;$ I2 = $26.5 \%$

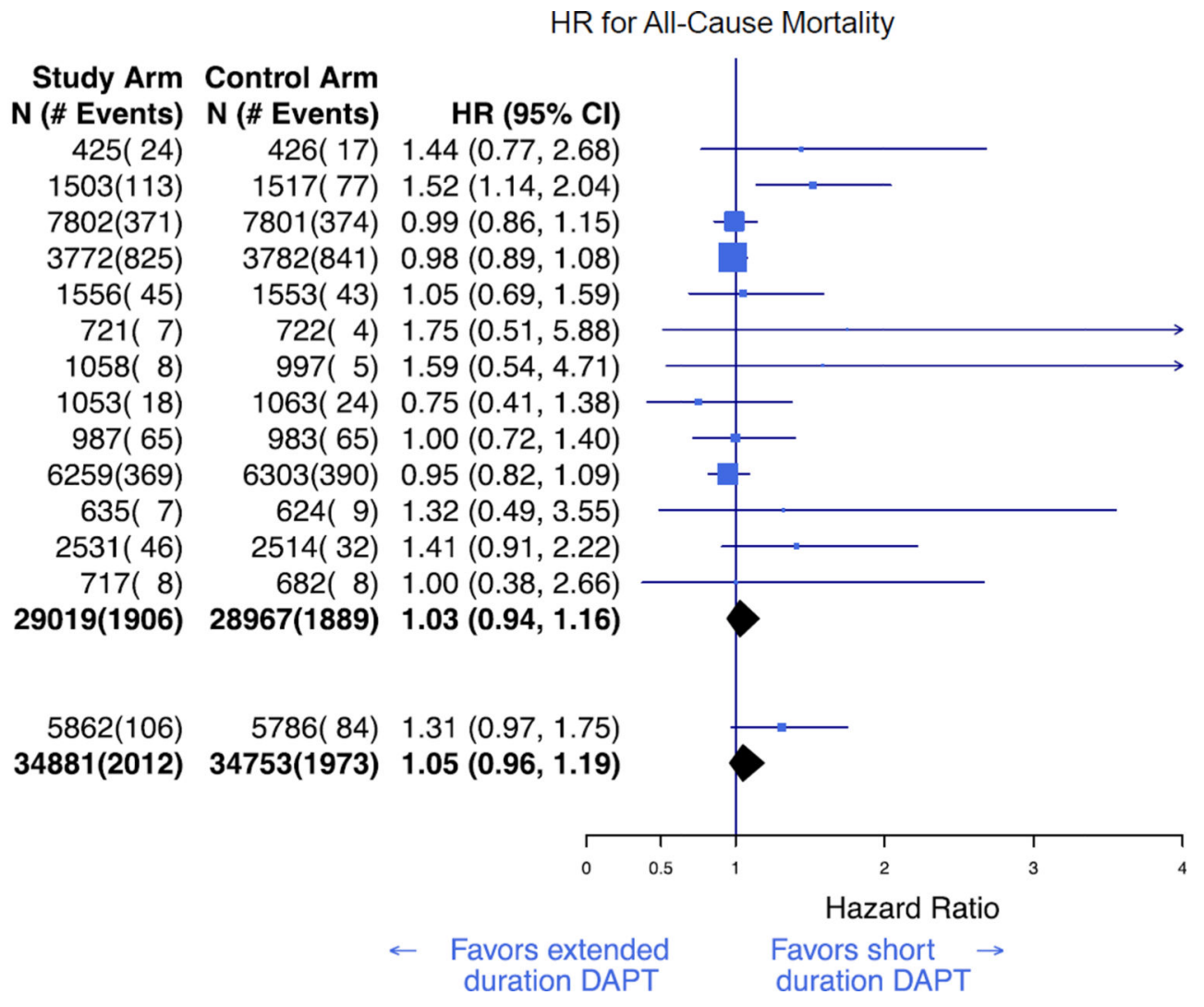

Figure 2. Bayesian meta-analysis of all-cause mortality associated with extended duration DAPT versus short duration or no DAPT

Results are presented prior to and after inclusion of the DAPT Study. ${ }^{3}$ DAPT=dual antiplatelet therapy. HR=hazard ratio. 
A.

Study
CASPAR
SPS3
CHARISMA
ACTIVE
OPTIMIZE
EXCELLENT
RESET
PRODIGY
CURE
DES LATE
SECURITY
Overall(DAPT NOT Included)
Q = 7.70, $p=0.66 ; 12=0.0 \%$
DAPT
Overall(DAPT Included)
Q $=7.70, p=0.74 ; 12=0.0 \%$

\section{HR for Cardiovascular Mortality}

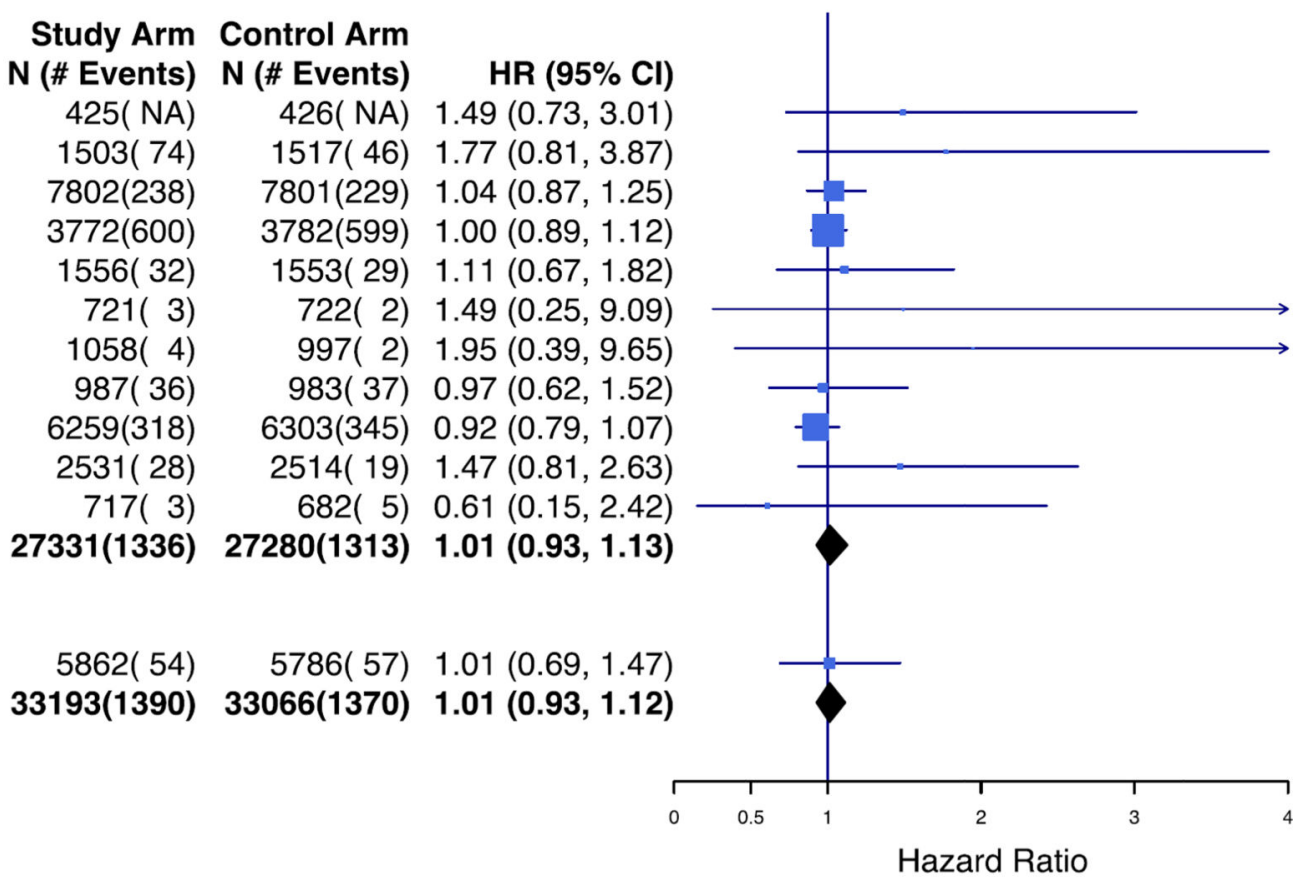

$\leftarrow$ Favors extended duration DAPT
Favors short $\rightarrow$ duration DAPT 
B.

Study
SPS3
CHARISMA
ACTIVE
OPTIMIZE
EXCELLENT
RESET
PRODIGY
CURE
DES LATE
SECURITY
Overall(DAPT NOT Included)
Q $=4.19, p=0.90 ; \mid 2=0 \%$
DAPT
Overall(DAPT Included)
Q = $11.61, p=0.31 ; 12=13.9 \%$

HR for Non-Cardiovascular Mortality

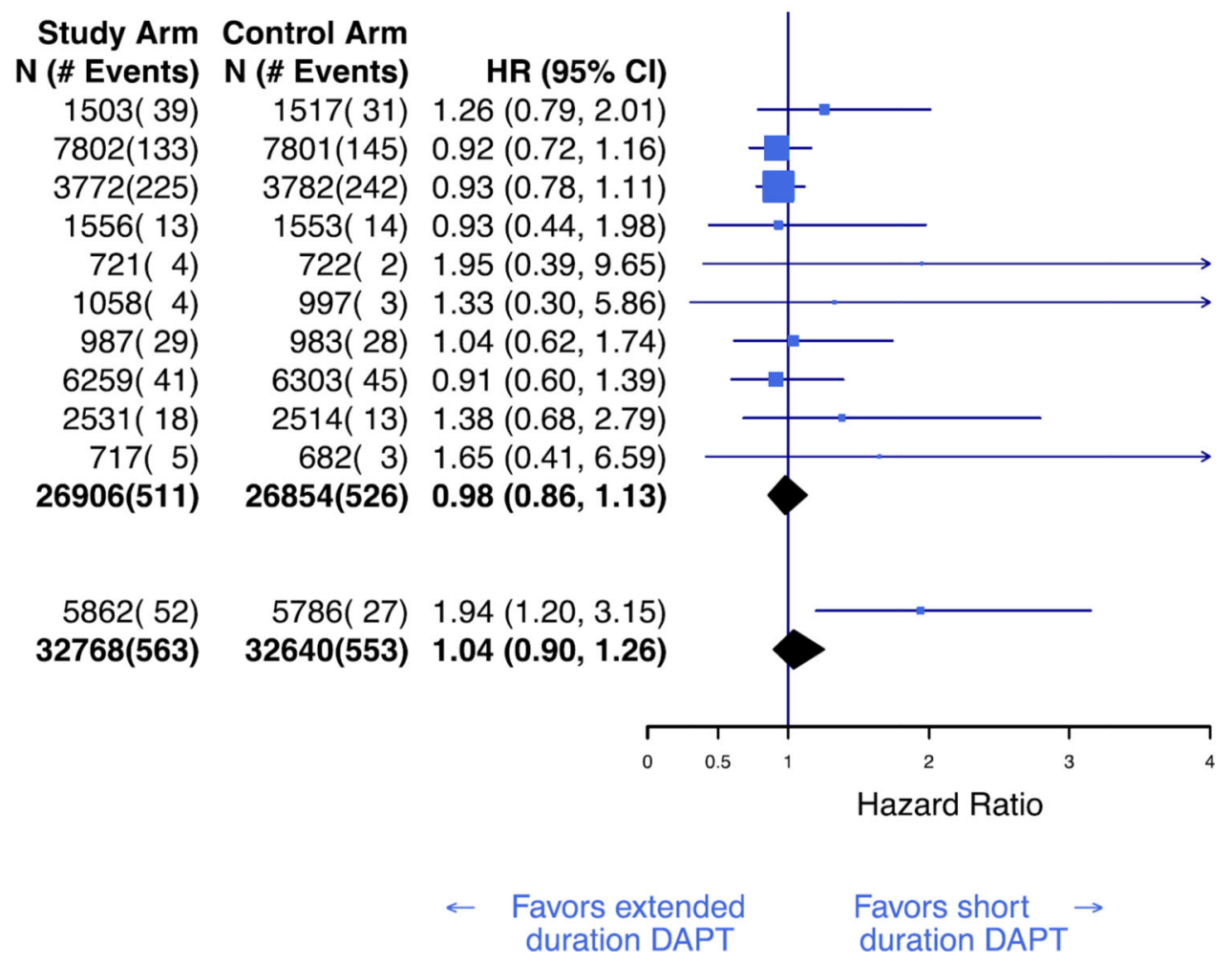

Figure 3. Bayesian meta-analysis of cardiovascular and non-cardiovascular mortality associated with extended duration DAPT versus short duration or no DAPT

Hazard ratio for cardiovascular mortality (A), and non-cardiovascular mortality (B). Results are presented prior to and after inclusion of the DAPT Study. ${ }^{3}$ The number of cardiovascular deaths in the CASPAR trial was not reported and is therefore not reflected in the total event count. ${ }^{11}$ DAPT=dual antiplatelet therapy. HR=hazard ratio. 


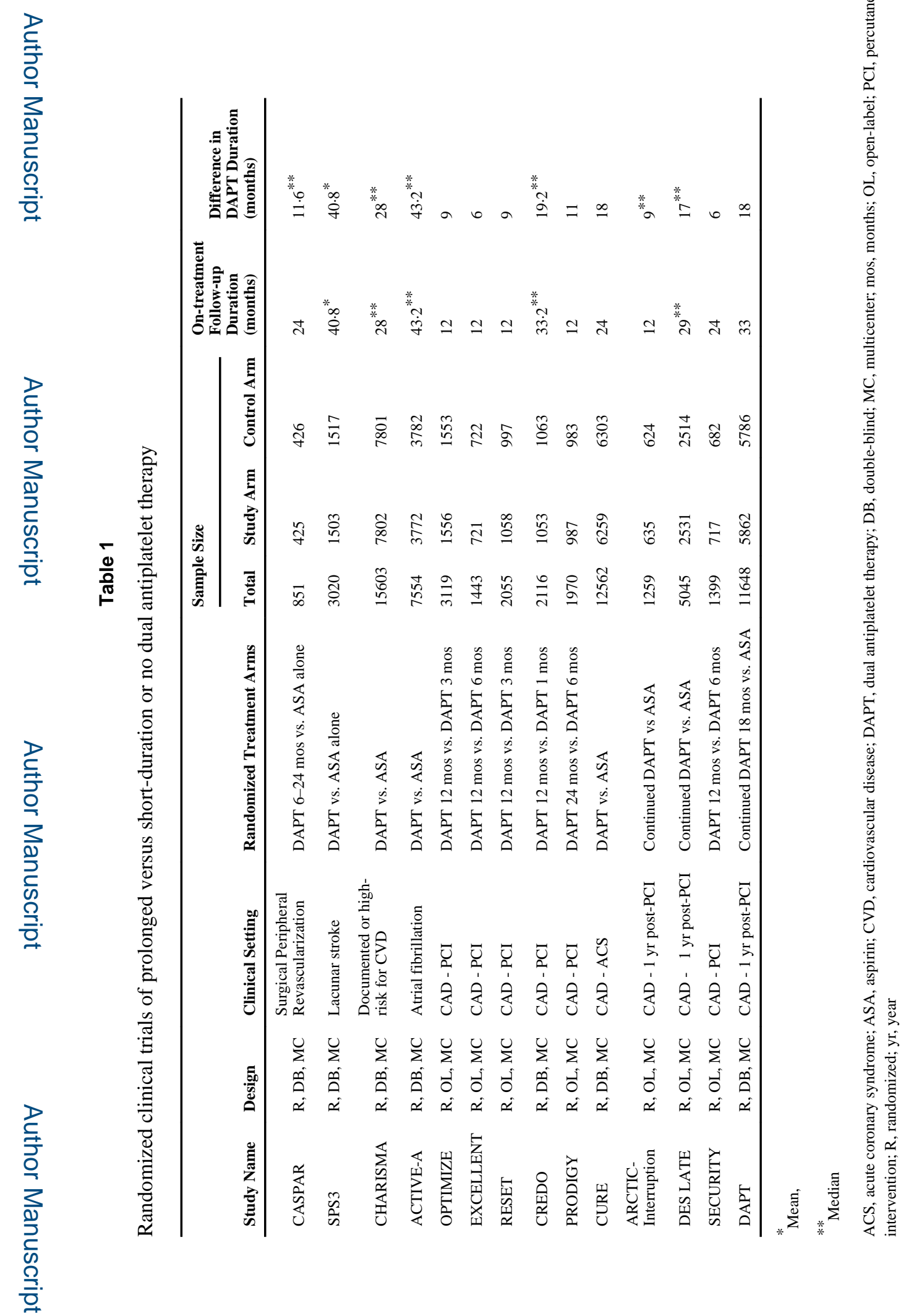

Lancet. Author manuscript; available in PMC 2016 February 28. 